Gerentes y equipo de trabajo efectivo: Empresa exitosa

\title{
Gerentes y equipo de trabajo efectivo: Empresa exitosa
}

\section{Managers and effective work team: successful company}

\section{Gerentes e equipe de trabalho eficaz: empresa de sucesso}

\author{
César A. Guerrero-Velástegui ${ }^{\mathrm{I}}$ \\ ca.guerrero@uta.edu.ec \\ Leonardo G. Ballesteros-López II \\ leonardogballesteros@uta.edu.ec \\ José B. Herrera-Herrera III \\ josebherrera@uta.edu.ec
}

Recibido: 14 de febrero de 2018 * Corregido: 25 de mayo de $2018 *$ Aceptado: 05 de junio de 2018

I. Magister en Administración de Empresas, Ingeniero de Empresas, Abogado, Docente Universidad Técnica de Ambato, Ambato, Ecuador.

II Magister en Gestión Estratégica Empresarial MBA, Ingeniero Comercial, Docente Universidad Técnica de Ambato, Ambato, Ecuador.

III Magister en Gestión Estratégica Empresarial MBA, Ingeniero de Empresas, Docente Universidad Técnica de Ambato, Ambato, Ecuador. 


\title{
Resumen
}

Los seres humanos buscan estar en grupos, en las organizaciones o empresas esa predisposición debe ser aprovechada para formar equipos de trabajo efectivos. Así han desarrollado distintos tipos de métodos para engranar efectivamente ese grupo. Entendiendo que debe haber una gerencia que supervise y represente esa empresa y sea quien tenga el contacto más cercano con el equipo y sea el responsable ante los dueños de los resultados. Los gerentes deben ser líderes, el ideal es el demócrata. Los equipos deben ser integrados por seres humanos con conocimientos académicos, como por aptitudes, Donde el grupo trabaje por el éxito individual, grupal y empresarial. Ese trabajo se hace con el manejo de roles, comunicación, compromiso y retribución. Cuando las personas deben trabajar en equipo, pero ser capaces de disentir, opinar y son respetados se pueden obtener mejores resultados. Las empresas exitosas han sabido formar y mantener esos equipos.

Palabras claves: equipo; trabajo; organización; líder; gerente; éxito; comunicación; clima; laboral; niveles.

\begin{abstract}
Human beings seek to be in groups, in organizations or companies that predisposition must be used to form effective work teams. Thus they have developed different types of methods to effectively engage that group. Understanding that there must be a management that supervises and represents that company and is the one who has the closest contact with the team and is responsible to the owners of the results. Managers must be leaders, the ideal is the democrat. The teams must be integrated by human beings with academic knowledge, such as skills, where the group works for individual, group and business success. This work is done with the management of roles, communication, commitment and retribution. When people must work as a team, but be able to disagree, give their opinion and be respected, better results can be obtained. Successful companies have been able to train and maintain these teams.
\end{abstract}

Keywords: equipment; job; organization; Leader; manager; success; communication; weather; labor; levels. 


\section{Resumo}

Os seres humanos procuram estar em grupos, em organizações ou empresas que a predisposição deve ser usada para formar equipes de trabalho eficazes. Assim, eles desenvolveram diferentes tipos de métodos para efetivamente envolver esse grupo. Entendendo que deve haver uma gerência que supervisiona e representa aquela empresa e é aquela que tem o contato mais próximo com a equipe e é responsável perante os proprietários dos resultados. Os gerentes devem ser líderes, o ideal é o democrata. As equipes devem ser integradas por seres humanos com conhecimento acadêmico, como habilidades, onde o grupo trabalha pelo sucesso individual, em grupo e empresarial. Este trabalho é feito com a gestão de papéis, comunicação, comprometimento e retribuição. Quando as pessoas precisam trabalhar em equipe, mas podem discordar, dar sua opinião e ser respeitadas, melhores resultados podem ser obtidos. Empresas de sucesso conseguiram treinar e manter essas equipes.

Palavras chave: equipamentos; trabalho; organização; líder gerente; sucesso comunicação; clima; trabalho níveis.

\section{Introducción}

Los seres humanos necesitan compañía de manera natural y por tanto tienden a buscar grupos donde integrarse, ya sean de tipo religioso, social, académico, etc. Esos grupos deben tener un propósito o finalidad.

Así, en los ambientes de trabajo se forman grupos que podemos llamar equipos de trabajo, cuyo objetivo es lograr buenos resultados. Cualquiera que sea la función o producto de la empresa $\mathrm{u}$ organización, el equipo de trabajo debe funcionar. Cada empresa debe buscar las estrategias más adecuadas para adaptarse al entorno y poder ser competitiva.

Las organizaciones o empresas deben aprender a seleccionar el personal que integre esos equipos de una manera armónica, desarrollando todo su potencial como individuos y grupo, para obtener los beneficios y producción que desean o buscan. Esos equipos deben tener un líder, que en las organizaciones se llama gerente, depende en la mayoría de los casos, según el tipo de gerente y el equipo que se forme para la calidad de trabajo que se desarrolle y el desarrollo del llamado sentido de pertenencia del empleado, por ello la empresa debe entender que su mayor capital es el humano. 
El presente artículo busca conocer y aprender cómo se forma ese equipo y sus relaciones con la organización a través de la gerencia, como mecanismo de éxito en las empresas.

\section{Metodología}

Se realiza investigación sobre qué es y cómo desarrollar una gerencia y trabajo en equipo; a través de revisión bibliográfica de artículos de revistas, trabajos de grado.

\section{Gerencia y gerentes}

Gerencia: es el proceso de hacer que las actividades sean terminadas con eficiencia y eficacia a través de otras personas. Es involucrar dentro de un proceso continuo y sistemático las acciones del talento humano, cuyo planteamiento sea realizar las actividades administrativas de forma óptima, controlada y con capacidad de resolver problemas. La gerencia debe trabajar para que la organización logre los mejores resultados. (Romero, 2005) (Cárdenas, 2000)

Gerentes: las personas que asumen el desempeño gerencial de una organización y son los responsables de dirigir las actividades que ayudan a las organizaciones para alcanzar sus metas, representando al empresario. Un buen gerente se conoce por la eficiencia y la eficacia que tenga. Es quien tiene la labor de cuidar, supervisar, controlar, planificar, las personas que están bajo su mando. Hay gerentes que entregan resultados y otros que son líderes y desarrollan personas, lo ideal es un gerente que sea capaz de las dos cosas. Debe saber mejorar las relaciones humanas en los grupos de trabajo y mantener un ambiente de trabajo interpersonal adecuado. El gerente debe fijar la visión, misión y valores y saberlos expresar al equipo para que sean aceptados. (Romero, 2005) (Carreño, 2017) (Cárdenas, 2000)

La fusión de gerente y líder es importante para las organizaciones productivas y así mantener altos nivel de eficacia y eficiencia. La finalidad es no solo dar órdenes, sino también saber delegar y ser capaz de realizar cambios cuando sean necesarios. (Ramírez, 2012)

\section{Se habla también de niveles de gerencia:}

Gerentes de Primera Línea: son los responsables del trabajo de los demás y son el nivel más bajo de la organización. Ejemplos: jefe o supervisor, director de escuela. 
Gerentes Medios: dirigen a los gerentes de primera línea y a empleados de operaciones, su responsabilidad es dirigir las actividades que ponen en práctica las políticas de la empresa y equilibran las demandas de sus gerentes y capacidades de los patrones.

Alta gerencia: menor número de personas y administran la organización, son ejecutivos. Ej: director general ejecutivo. (Barrios, 1999)

\section{Equipo de trabajo}

\section{Para hablar del equipo, leamos definiciones de grupo:}

Grupo es un conjunto de personas que interactúan directamente entre sí, reunidas para alcanzar un objetivo común y que llegan a integrar una formación relativamente estable en el tiempo con una determinada estructura (roles y normas grupales) y procesos dinámicos internos. (Chibás, 1992)

Otra definición de grupo: como "un conjunto restringido de personas que, ligadas entre sí por constantes de tiempo y espacio, y articuladas por su mutua representación interna se proponen en forma explícita e implícita una tarea que constituye su finalidad, interactuando la adopción y adjudicación de roles". (Bleger, 1961)

Las personas tienen tres necesidades personales, que pueden estar combinadas, pero predominando una de ellas:

Necesidad de logro, su motivación es lograr algo difícil, mediante el reto y desafío de sus propias metas y con ello avanzar en el trabajo. Estas personas poseen una fuerte necesidad de retroalimentarse de su logro y progreso, así como una necesidad por sentirse dotado, realizado, gratificado y con talento.

Necesidad de poder y autoridad, su motivación está en influir, adiestrar, enseñar o animar a los demás a conseguir logros. Quieren que sus ideas prevalezcan y buscan aumentar su poder.

Necesidad de asociación o afiliación, su motivación es tener relaciones amigables y de interacción con la gente y con los demás compañeros de trabajo. La afiliación conduce a sentirse respaldado por la ayuda, respeto y consideración de los demás. No son los líderes ni los directivos más eficientes, ya 
que les cuesta mucho tomar decisiones difíciles sin preocuparse por disgustar a los demás. (Tortolero, 2015)

Identificar en los empleados estas necesidades, ayuda a ubicar su posición en el equipo de trabajo.

La selección de las personas que integran ese equipo debe ser realizada acorde a las competencias individuales, identificando sus necesidades y funciones del cargo que se le va asignar, además deben saber trabajar en equipo.

\section{Por ello hay que hablar de distintos puntos:}

Rol: es acorde a los conocimientos, experiencia, habilidades que cada uno tenga. Los individuos deben percibir que se les delega autoridad y pueden desempeñar sus trabajos sin tener que consultar constantemente al supervisor. (blog.dacompanama.com, 16)

Comunicación: es la acción y efecto de comunicar o comunicarse, es la transmisión de un mensaje entre un emisor y un receptor mediante un código común. Los filósofos, sociólogos y psicólogos, por su parte, se han servido de este concepto para designar el conjunto de modos específicos que pueden adoptar las relaciones humanas en cuanto son, o pueden ser, relaciones de participación recíproca o de comprensión de los seres humanos que participan en la misma. (Parra de Párraga, Rojas, \& Arapé, 2008) (blog.dacompanama.com, 16)

En las organizaciones la comunicación abarca los impresos, folletos internos, memorandos, charlas, así como el sentido de pertenencia, nivel de satisfacción, beneficios y el ejemplo de los jefes. La comunicación también se puede considerar, vertical (puede ser descendente o ascendente) cuando fluye de un nivel administrativo superior, a uno inferior, o viceversa: quejas, reportes, órdenes e instrucciones. Es horizontal: cuando es entre niveles jerárquicos semejantes: memorándum, circulares y juntas entre otros. Se usa para integrar y organizar funciones. Además, está la verbal y la escrita. (Tortolero, 2015) (Parra de Párraga, Rojas, \& Arapé, 2008)

La comunicación es indispensable para informar a los integrantes del equipo sobre qué sucede, que se expresen acuerdos y desacuerdos sobre el trabajo. Se deben conocer las restricciones o flexibilidad en la organización, es decir, hasta qué punto las reglas, políticas, procedimientos o prácticas son innecesarias o interfieren con la ejecución del trabajo. Todo ese intercambio de información, ayudaa 
Gerentes y equipo de trabajo efectivo: Empresa exitosa

generar conocimientos y aprendizaje. (López Duque, Lanzas Duque, \& Lanzas Duque, 2007) (blog.dacompanama.com, 16) (Muñoz, 2014)

Liderazgo: el líder es quien permite motivar al tener claro el objetivo y es efectivo al transmitirlo a los otros integrantes, para que tengan el interés de realizar bien y mejorar su trabajo, incentiva la creatividad y motiva convenientemente. El buen líder desarrolla personas. Debe existir respeto y confianza más que miedo y sumisión hacia el líder. Hay gerentes líderes y otros no, en el liderazgo el poder no es por la posición, esta concedido por el grupo. (Tortolero, 2015) (Ferrer, Clemenza, Romero, \& Rojas, 2000) (Carreño, 2017) (Cárdenas, 2000) (blog.dacompanama.com, 16)

Warren Benis definió diez características personales y organizacionales que son necesarias en un líder, para forjar el futuro: tener un sueño o visión de futuro, tomar riesgos, acompañarse de crítica, ser optimista, estimular el disentimiento, tener expectativas altas frente a los demás, tener olfato sobre el futuro, ver las cosas a largo plazo, comprender y respetar los intereses creados, y desarrollar alianzas estratégicas. (Bennis, 1989).

En el liderazgo se puede describir tres tipos: autoritario o autocrático, democrático y laissez faire.

El autoritario: trata a los integrantes del equipo como inferiores, desestima ideas o sugerencias; muestra trato preferencial hacia algunos miembros; ese comportamiento no es adecuado, provoca agresividad o apatía, frustración. El democrático: estimula las relaciones entre sus integrantes, hay mayor compromiso y agrado al realizar el trabajo. Este líder favorece las discusiones, hay menor tensión, permite la discusión de cómo hacer la tarea, busca el consenso. El líder laissez practica una especie de abandono de autoridad, no se interesa por la actividad de grupo, se deja desbordar por él, otorga recompensas y castigos en forma arbitraria, no se compromete con el grupo, solo está presente para que el grupo pueda recurrir a él en caso que lo necesite. (Luptáková)

Para lograr una organización productiva debe prevalecer el líder democrático.

Compensación: es importante para que los integrantes sepan que su esfuerzo es reconocido; la compensación económica es importante, pero las felicitaciones también son un estímulo para mantener el interés del equipo y por tanto su compromiso. (blog.dacompanama.com, 16) 
Compromiso: al establecer un vínculo entre los integrantes del equipo, se identifican sus propósitos y cada quien ejerce su rol se genera el sentido de pertenencia para hacer que el equipo se comprometa en logar el éxito u objetivo, al tener sentido de pertenencia al equipo y a la empresa. También, el grado en que la gente se siente orgullosa de pertenecer a la organización y sienten que todos están trabajando hacia un objetivo común. (López Duque, Lanzas Duque, \& Lanzas Duque, 2007) (blog.dacompanama.com, 16)

Conflicto: es parte de nuestras vidas, debemos aprender a enfrentarlo de la manera adecuada; se presenta cuando hay desacuerdo y sendas partes se radicalizan, por lo cual deben usar la comunicación para lograr una salida, actuando con respeto a las reglas de juego. (Parra de Párraga, Rojas, \& Arapé, 2008)

\section{Ventajas del trabajo en equipo:}

Permite que sea más fácil la consecución de objetivos.

Contribuye al mejoramiento de la calidad de vida de los miembros.

Agrega valor a los procesos.

Cada integrante puede poner al servicio del equipo sus competencias individuales.

Las discrepancias bien discutidas, llevaran a crear soluciones distintas.

Permite compartir las metas y objetivos del trabajo.

Disminuye rotación de personal al trabajar en un lugar agradable.

\section{Desventajas del trabajo en equipo:}

El tener que conocer e interrelacionarse con otros se convierte en ocasiones en fuente de conflicto para algunos de los integrantes.

En ocasiones puede diluirse la responsabilidad personal al tomar al equipo como escudo frente a los resultados negativos. 
Se dan problemas de comunicación y falta de asertividad que dificulta el avance del equipo.

Un equipo bien engranado está conformado por personas que saben para que, y porque están en el equipo, tienen interés, trabajan por lograr la meta, apoyan a sus compañeros, se alegran de los éxitos individuales y reconocen sus fracasos, todo para lograr aprendizaje y mejorar la capacidad de desarrollo individual y grupal.

Otra manera de plantear el equipo de trabajo exitoso es a través de la definición de un clima psicosocial:

“Conjunto de características objetivas de la organización, perdurables y fácilmente medibles, que distinguen una entidad laboral de otra. Son unos estilos de dirección, unas normas y medio ambiente fisiológico, unas finalidades y unos procesos de contraprestación. Aunque en su medida se hace intervenir la percepción individual, lo fundamental son unos índices de dichas características". (Forehand y Gilmer, 1965) (areaRH.com)

"Conjunto de percepciones globales (constructo personal y psicológico) que el individuo tiene de la organización, reflejo de la interacción entre ambos; lo importante es cómo percibe un sujeto su entorno, sin tener en cuenta cómo lo percibe otros; por tanto, es más una dimensión del individuo que de la organización". (areaRH.com)

El equilibrio de una organización requiere liderazgo y compromiso de todos sus miembros con los objetivos y metas que ésta se ha trazado en un tiempo y espacio determinado, aun cuando se presenten emergencias. (Ramírez, 2012) (areaRH.com)

En este cuadro también podemos ver cómo se desarrolla el potencial del equipo de trabajo 


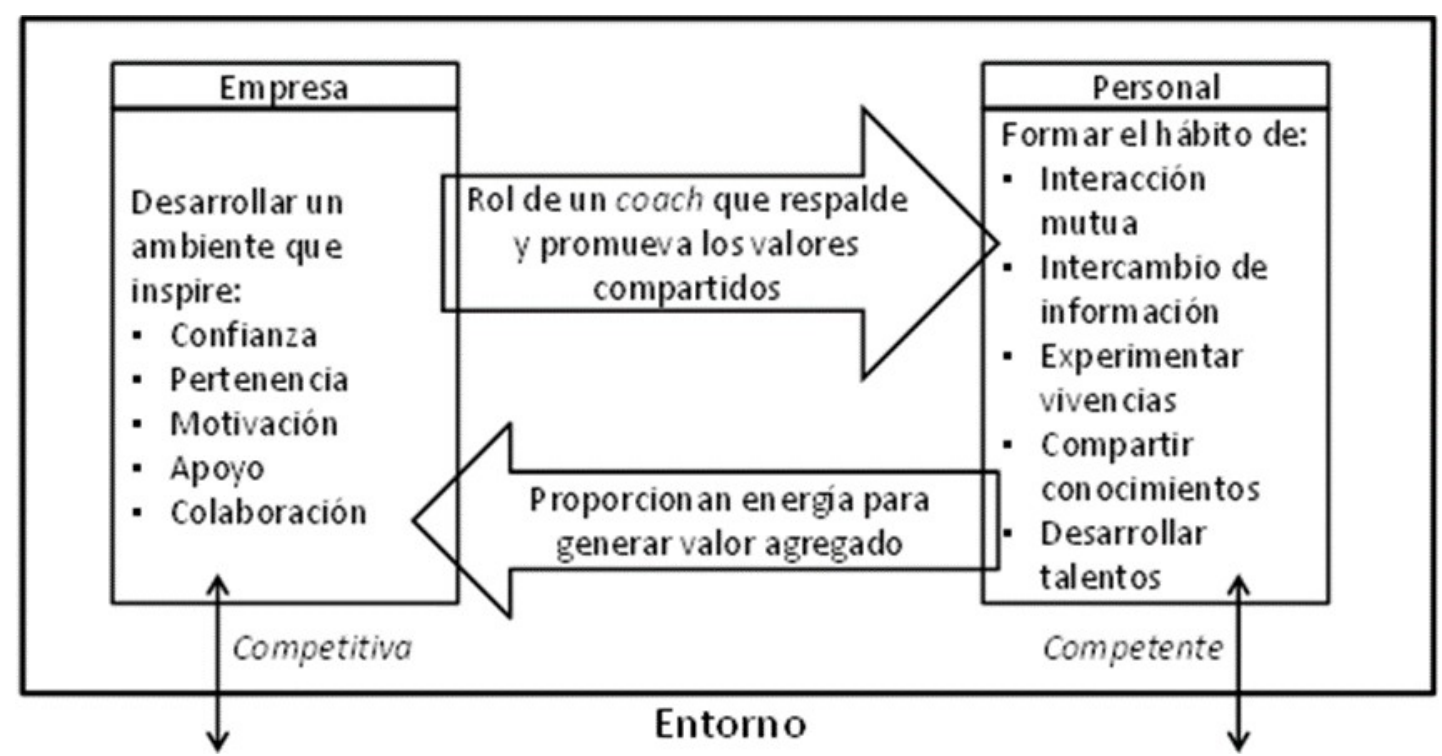

..." El cuadro anterior ilustra la necesidad de una empresa de desarrollar un ambiente que se caracterice por respaldar y promover: confianza, sentido de pertenencia, motivación, apoyo y colaboración, con lo que podrá facilitar el hábito entre sus empleados, de interactuar, comunicar y dialogar, sobre sus vivencias y experiencias de trabajo, además de intercambiar y compartir conocimientos con los que contribuirán a desarrollar competencias y talentos en su ámbito laboral..." (Hernández, 2015)

... "La interconexión de ambas partes, será el personal de mando, quienes deben asumir el rol de coach para guiar y entrenar a los colaboradores, y ser también el canalizador de la energía de los talentos del personal a generar el valor agregado con que la empresa logrará sus propósitos. Estos elementos contribuirán a que la empresa sea competitiva y el personal competente, para responder al entorno que se ven inmiscuidos. Como puede observarse, desarrollar una cultura orientada al aprendizaje, requiere de mayor atención hacia el trabajo colaborativo con los miembros que integran equipos; apertura muestras de confianza y diálogo, con las que se perciba un clima que contribuya al intercambio de conocimientos, ideas y experiencias que cognitivamente tiene cada empleado $\mathrm{y}$, mediante procesos de comunicación, intercambia y enriquece con sus compañeros.” ... (Hernández, 2015) 
Gerentes y equipo de trabajo efectivo: Empresa exitosa

\begin{tabular}{l|c|l|}
\hline Unidad de competencias & Página & \multicolumn{1}{c|}{ Competencias } \\
\hline Comunicación & $26-27$ & $\begin{array}{l}\text { Escuchar activamente, entender y dar respuesta a interesados, } \\
\text { mantener líneas de comunicación, asegurar la calidad de la } \\
\text { información, adaptar comunicaciones. }\end{array}$ \\
\hline Liderazgo & $28-29$ & $\begin{array}{l}\text { Crear ambiente de trabajo en equipo, construir y mantener relaciones } \\
\text { efectivas, motivar y dar tutoría a los miembros del equipo, asumir } \\
\text { responsabilidad por los entregables, usar herramientas para influir. }\end{array}$ \\
\hline Gerencia & $30-31$ & $\begin{array}{l}\text { Construir y mantener el equipo del proyecto, planear y manejar el } \\
\text { desarrollo del proyecto, resolver conflictos. }\end{array}$ \\
\hline Habilidades cognitivas & $32-33$ & $\begin{array}{l}\text { Desarrollar una visión holística del proyecto, solucionar efectivamente } \\
\text { problemas, utilizar apropiadamente herramientas y técnicas de } \\
\text { gerencia de proyectos, buscar oportunidades para mejorar los } \\
\text { resultados del proyecto. }\end{array}$ \\
\hline Efectividad & $34-35$ & $\begin{array}{l}\text { Resolver problemas, mantener motivación, compromiso y apoyo de } \\
\text { los interesados Actuar oportunamente frente a los cambios, usar } \\
\text { asertividad. }\end{array}$ \\
\hline & $36-33$ & $\begin{array}{l}\text { Demostrar compromiso con el proyecto, operar con integridad, } \\
\text { manejar las adversidades del personal del equipo, dirigir fuerzas de } \\
\text { trabajo diversas, solucionar problemas individuales y organizacionales. }\end{array}$ \\
\hline
\end{tabular}

Unidades de competencias personales según el Project Management Competency Development Framework, 2da edición. (Carreño, 2017)

\section{Revisión de trabajos de investigación:}

Una empresa multinacional de gran consumo (estudio internacional HayMcBer, 1993-95). Un estudio de directivos de una empresa multinacional de productos de gran consumo demostró la relación entre el clima positivo y los indicadores de desempeño. A principios del año fiscal, se entregó a cada directivo unos objetivos financieros para su unidad. La retribución variable del directivo y de la de su equipo estaba directamente relacionada con esta medida del desempeño. A finales del año fiscal, mostró una correlación positiva entre las dimensiones del clima y las medidas de desempeño. Es decir, independiente del país donde la empresa desarrollará su negocio, las unidades que mostraron mejor clima lograron mejores resultados financieros. Una empresa multinacional petroquímica (estudio EEUU, HayMcBear, 1993). Se llevó a cabo un estudio de las tres principales unidades de negocio para identificar oportunidades de desarrollo. Se midió y comparó el clima de aproximadamente 350 directivos en varias unidades de negocio en cuanto a dos variables de desempeño clave: cash flow e ingresos operativos netos. Las dimensiones del clima mostraron una fuerte correlación con ambas variables, ya que las unidades con mejor clima resultaron ser las que mejores resultados financieros 
Gerentes y equipo de trabajo efectivo: Empresa exitosa

obtuvieron y las que mostraron peor clima, consecuentemente lograron peores resultados de negocio. LOMA CEOs (estudio realizado en Estados Unidos, 1994 HayMcBear). En un estudio de los presidentes de una compañía líder del sector asegurador, el clima que estos directivos generaban en sus colaboradores era predictivo del desempeño de la organización en general. Se dividió la compañía en dos grupos: los que mostraron un clima positivo y los que mostraron un clima promedio. En este estudio, se comprobó que la variable de clima era predictiva de altos o bajos niveles de desempeño en un 69 por 100. En el caso de variables específicas, la precisión aumentó a un 75 por 100. (Falcon, 2005)

Con respecto a las mujeres, se evidencia el aumento en el número de mujeres que se forman y se vinculan a grupos de interés relacionados con el tema. Según el International Business Report (Grant Thornton, 2014), globalmente, solo $24 \%$ de los cargos gerenciales son ocupados por mujeres, y en países como Alemania, solo $33 \%$ de las empresas cuenta con mujeres en posiciones gerenciales. Los hombres siguen predominando en gerencia de proyectos, especialmente en ingeniería, construcción, informática, telecomunicaciones y proyectos aeroespaciales, entre otros. (Botkova, 2008)

Dentro de la revisión se encontró un trabajo que habla del papel de las mujeres en cargos de funcionarios y gerentes en México. Así reporta lo siguiente:

El porcentaje se incrementó del 10\% en 1991 al 24,8\% en 2006. Pero, como sucede en otros países, a medida que se asciende en la pirámide organizacional, el número de mujeres disminuye significativamente. Con base en los datos de las 500 empresas más importantes de México, se ha podido demostrar que, a pesar del creciente número de compañías que tienen por lo menos una mujer en los altos cargos directivos, su porcentaje en estos apenas representa el 13\% y sólo se encuentran cuatro mujeres $(0,9 \%)$ como directoras generales (CEO).

Las direcciones operativas continúan siendo "territorio masculino" y las ejecutivas se concentran en las direcciones de recursos humanos, relaciones públicas y mercadotecnia. De hecho, se encontró que el incremento de la participación de las mujeres en las altas esferas se explica en gran parte por la creciente incorporación en estas dos últimas áreas que antes no aparecían como direcciones generales dentro de los organigramas de muchas compañías. 
Hay mayores probabilidades de encontrar ejecutivas en las empresas más pequeñas con estructuras más flexibles y menos burocráticas y en las compañías de capital extranjero. La situación con respecto a estas últimas puede tener parte de su explicación en el hecho de que durante los últimos años las filiales mexicanas han puesto en marcha algunas políticas de diversidad que antes sólo existían en las matrices.

La brecha entre la presencia masculina y la femenina en los cargos de dirección se explica por factores socioculturales de carácter general que conforman gran parte de las personalidades y expectativas sobre los hombres y mujeres en el ámbito de lo público y lo privado.

Así, el notable incremento de la participación de las mujeres en el empleo remunerado y la existencia de prácticas más coparticipativas en el ingreso familiar no se han visto acompañadas de un replanteamiento de las responsabilidades de los hombres dentro del hogar. (Zabludovsky, 2007)

En Colombia, se realizó un cuestionario a 21 mujeres gerentes de proyecto, que reportó los siguientes resultados: $52 \%$ de las mujeres tenían especialización, $24 \%$ maestría y $5 \%$ doctorado. De ellas 38 $\%$ cuentan con especialización en el área de proyectos, pero $24 \%$ no cuenta con formación específica en el tema. Las mujeres que respondieron la encuesta muestran, de acuerdo con los resultados, que han desarrollado su vida familiar en condiciones relativamente normales, a pesar de sus compromisos por el rol gerencial, $48 \%$ de ellas están casadas con hijos, y de las solteras $43 \%$ tiene menos de 33 años. Con respecto a los ingresos, el 57\% se encuentran en rangos inferiores al valor promedio; de la muestra el $33 \%$ considera que los hombres ganan más. (Carreño, 2017).

\section{Conclusiones}

Luego de la revisión, se entiende que una organización productiva es consecuencia de un equipo bien integrado con líderes efectivos. Las empresas deben dedicar esfuerzos para seleccionar a los empleados tomando en cuenta la parte académica, sin olvidar las aptitudes individuales. Los equipos de trabajo se integran según las necesidades y logros de cada individuo, formando un grupo que busca el éxito, individual, grupal y empresarial.

Hay múltiples maneras y métodos para formar ese equipo, pero todas concluyen o tiene en común que la integración efectiva se logra: informando, escuchando, respetando, con compromiso y retribuciones efectivas en lo económico como en lo personal. 
El mayor estimulo es la comunicación efectiva, es decir, que fluya en ambas direcciones.

Entender que no siempre el gerente es el líder: aun cuando es lo ideal que combine ambas posiciones. Cada empresa u organización debe buscar y trabajar o desarrollar el método más efectivo para lograr ese equipo de trabajo.

Las mujeres siguen siendo minoría en los cargos de gerentes, lo cual debe ser evaluado.

Las empresas exitosas han entendido que el capital más importante en toda organización es el ser humano.

\section{Referencias Bibliográficas}

AreaRH.com. (S.f.). Recuperado el 31 de mayo de 2018, de www.arearh.com/rrhh/definicionclima.htm

Barrios, G. (julio de 1999). Gerencia y liderazgo. Venezuela: www.monografias.com.

Bleger, J. (1961). Grupos operativos en la enseñanza. Conferencia dictada en la Asociación de Psicología y Psicoterapia de Grupo. Buenos aires, Argentina.

blog.dacompanama.com. (10 de 10 de 16). Recuperado el 30 de mayo de 2018, de http://www.degrencia.com/articulo/importancia_del_trabajo_en_equipo_en_la_organziacion

Botkova, Z. (2008). The Progression of Women in Project Management. Middlesex University.

Cardenas, R. (2000). GERENCIA BASADA EN EL LIDERAZGO. San Nicolás de las Garzas, México: UNIVERSIDAD AUTONOMA DE NUEVO LEON.

Carreño, F. V. (2017). Caracterización del rol actual de la mujer en la gerencia de proyectos en Colombia. Revista EAN, 93-117.

Chibás, F. (1992). Creatividad, más dinámica de grupo. Habana: Pueblo y Educación.

Falcon, E. (23 de noviembre de 2005). monografias.com. Recuperado el 31 de 05 de 2018, de www.monografias.com/trabajos 31 
Ferrer, J., Clemenza, C., Romero, D., \& Rojas, L. R. (2000). Liderazgo Corporativo y Crisis Paradigmática Universitaria. Convergencia. Revista de Ciencias Sociales, vol. 7, núm. 23, septiembre, 2000, 151-172.

Hernández, N. C. (19 de 10 de 2015). La cultura y clima organizacional orientadas al aprendizaje en una empresa competitiva. Recuperado el 3 de junio de 2018, de deGerencia.com: http://www.degerencia.com/

López Duque, E., Lanzas Duque, A. M., \& Lanzas Duque, V. E. (2007). Administración del cambio en las organizaciones. Scientia Et Technica, vol. XIII, 301-303.

Luptáková, M. F. (s.f.). EL TRABAJO EN EQUIP O: UNA ALTERNATIVA PARA EL CAMBIO DE LA CULTURA INSTITUCIONAL. Educare.

Muñoz, P. (2014). EL SENTIDO DE PERTENENCIA COMO ESTRATEGIA ORGANIZACIONAL PARA EL APORTE DE LOS OBJETIVOS DE LA EMPRESA. Nueva Granada: UNIVERSIDAD MILITAR NUEVA GRANADA.

Parra de Párraga, E., Rojas, L. R., \& Arapé, E. (2008). Comunicación y conflicto: el arte de la negociación. Negotium, vol. 4, núm. 10, 17-35.

Ramírez, M. (2012). Estilos de liderazgo y sus enfoques gerenciales. Una aproximación teóricametodológica para el análisis de la dirección organizacional. Revista de Ciencias Sociales (Ve), vol. XVIII, núm. 1, enero-marzo, 2012, pp. 89- 98, 89-98.

Romero, G. E. (2005). Papel del gerente líder venezolano: Gerencia vs. Liderazgo. Telos, vol. 7, 51 65.

Tortolero, N. (abril de 2015). LIDERAZGO Y GERENCIA EFECTIVA EN ELC.E.I. GUSTAVO H MACHADO. Barbula, Carabobo, Venezuela: UNIVERSIDAD DE CARABOBO.

Zabludovsky, G. (2007). México: mujeres en cargos de dirección del sector privado. Academia. Revista Latinoamericana de Administración., 9-26. 